\title{
Tight junction protein claudin 3 expression in glioma
}

\author{
Hanuma Kumar ${ }^{1}$, BSA Raju ${ }^{2}$ Nagaiah $\mathrm{K}^{1}$ \\ ${ }^{1}$ (Biotechnology Dept/ GP Limited, Hyderabad, India) \\ ${ }^{2}$ (Tumor Biology Dept, Institute of Pathology, New Delhi India) \\ ${ }^{3}$ (Biotechnology Dept/ GP Limited, Hyderabad, India)
}

\begin{abstract}
Among the brain tumors, Glioma is the most malignant disease that causes death and the prognosis of patients remains poor. Tight junctions (TJs) are important in formation of endothelial cells of the blood-brain barrier (BBB) and acts as a restrictive paracellular diffusion barrier. Claudins are imp components of TJs, and expression of these proteins were altered selectively in cerebral microvessels of human GBM and other pathological conditions, resulting in brain edema. The aim of this study was to determine the pattern of expression of tight junction associated protein claudin 3 in low grade and high grade glioma cell lines C6, U373, U118, T98 and U87MG using Real Time PCR and western blot methods. Interestingly quantitative Real Time PCR and western blot results have shown down regulation of claudin 3 in high grade glioma to low grade glioma. Our results concluded that decreased expression of claudin 3 was correlated with disease progression.

Key words: Glioma, Tight junction, Claudin 3 and Blood Brain Barrier
\end{abstract}

\section{INTRODUCTION}

Glioblastoma multiforme is the most malignant disease among the brain tumors and is well characterized by pronounced hypercellularity, pleomorphism, numerous mitoses, foci of necrosis and palisading, and excessive vascularization with morphological alterations in blood vessels [1-3]. About $80 \%$ of malignant primary brain tumors are gliomas. Although brain tumors can occur at any age, they are most common in children of 3 to 12 years age and in adults of 40 to 70 years age. The majority of patients with this grade of tumor do not respond to surgical resection, radiation therapy and adjuvant chemotherapy. Achievement of effective new treatments can be possible only through better understanding of the molecular mechanism of disease.

BBB protects brain from a changing composition of the blood and restrict the paracellular diffusion of hydrophilic molecules due to highly specialized endothelial cells which contain an elaborate network of complex tight junctions (TJs) between the endothelial cells $(4,5)$. In glioma and other pathological conditions of brain, blood vessels lose their BBB nature, which results in severe edema [6].

In recent years, reports have shown tight junction proteins were associated with epithelial and endothelial cells. TJ-associated proteins include cytoplasmic peripheral membrane proteins and integral membrane proteins. Cytoplasmic peripheral membrane proteins ZO-1 and ZO-2 belongs to MAGUK family [7] and Integral membrane proteins occluding $[8,9]$ and the claudins, which comprise a novel gene family of four transmembrane TJ proteins with no sequence homology to occludin $[10,11]$.

The experiment of transfection of fibroblasts with claudins induced in absence of occludin, proved that claudins are important for $\mathrm{TJ}$ induction. To date, 24 members of the claudin family proteins with different tissue distribution have been described [20]. In the CNS, claudin- 1 and claudin- 5 have been detected in BBB endothelium at the protein level [12-14]. Furuse et al. [10] described in a Northern blot analysis the expression of claudin-1 and the absence of claudin-2 in whole brain preparations [15].

In the present study we investigated expression of claudin 3 transcription levels in low grade and high grade glioma cell lines. Real Time PCR and western blot methods were employed to asses mRNA expression levels. In this study we have used cell lines C6, U373, U87 MG, U118 and T98, and ECV 304 was used as control. The current study suggested that a down regulation of blood brain barrier component claudin 3 was observed in high grade cell lines.

\subsection{Glioma Cell cultures}

\section{MATERIALS AND METHODS}

The malignant glioma cell lines comprising C6 (lower grade), U118 and U373, T98MG, U87MG (higher grade) in and control cell line ECV304, used in the current study. All the cell lines were maintained in Dulbecco's modified Eagle's media [DMEM] with supplementations of $10 \%$ heat-inactivated fetal bovine serum, $2 \mathrm{mM} \mathrm{L}$-glutamine, $50 \mathrm{U} / \mathrm{mL}$ penicillin, and $50 \mu \mathrm{g} / \mathrm{mL}$ streptomycin. All the cell lines were grown up to $80 \%$ confluence in $75 \mathrm{~cm}^{2}$ culture flasks at $37^{\circ} \mathrm{C}$ in incubator with $5 \% \mathrm{Co} 2$. Cell line sub culturing was done for 48 hours trypsinization method, centrifuging and resuspending in fresh DMEM. Finally, the cultured cells were 
scraped from culture flasks and single cell suspensions were prepared in TRIZOL reagent (Invitrogen) by passing pieces of cells through series of sequentially smaller hypodermic needles (22-30 gauge) and stored at $80^{\circ} \mathrm{c}$ until processed further for RNA and protein isolations.

\subsection{Cell viability assays}

The viability of cultured was determined by standard tryphan blue exclusion test, where $10 \mu$ trypan blue solutions was added to $10 \mu \mathrm{l}$ cell suspension containing approximately $2 \times 10^{4}$ cells at logarithmic growth phase. The counting scores of stained and unstained cells on hemocytometer were based to estimate the cell survival.

\subsection{RNA extractions and Real-time polymerase chain reaction analysis}

Total RNA from cultured cells (approximately $10^{6}$ cells) was extracted using TRIZOL reagent (Invitrogen) according to manufacturer's instructions. After DNaseI treatment, all RNAs were reversetranscribed into cDNAs with Superscript II reverse transcriptase (Invitrogen, USA) and oligo (dT) primers in accordance to the manufacturer's protocol instructions. The specific mRNA quantities of claudin 3 in all cDNA samples were determined by Real-time reverse transcriptase (RT)-polymerase chain reaction (PCR) method using ABI Prism 7000 Sequence Detection System. Each $20 \mu$ reaction mixture contained $12.5 \mu \mathrm{l}$ of $2 \mathrm{X}$ Power SYBR Green PCR Master Mix (Applied Biosystems), $5 \mu \mathrm{l}$ of cDNA, and $10 \mathrm{pm}$ primer pairs (listed in Table 1). To counterbalance variations in PCR efficiency, standard curve analysis with serially diluted pooled cDNAs was done for primer sets in each reaction set up. PCR reaction conditions included 2 mins at $95^{\circ} \mathrm{C}$ for initial denaturing, then 40 cycles of $95^{\circ} \mathrm{C}$ for $20 \mathrm{~s}, 63^{\circ} \mathrm{C}$ for $30 \mathrm{~s}$, and $72^{\circ} \mathrm{C}$ for $30 \mathrm{~s}$ annealing, followed by melting analyses from 55 to $95^{\circ} \mathrm{C}$. RT-PCR reactions of claudin 3 for each sample were done in triplicates in 96-well plates. Melting curves were checked to verify the melting temperatures of PCR amplicons. Additionally, PCR amplicons from the real-time master plate were subjected to electrophoresis on $2 \%$ agarose gel to confirm the success of PCR reaction. GAPDH was used as reference gene. The relative expression levels for claudin 3 was estimated according to the $\Delta \Delta \mathrm{Ct}$ approximation method by normalizing estimates of claudin 3 to GAPDH levels $[\mathrm{XN}=2(-\Delta \mathrm{Ct})$, where $\Delta \mathrm{Ct}=(\mathrm{Ct}$ of CDLN3 $-\mathrm{Ct}$ of GAPDH $)]$. The normalized levels of the transcripts in glioma cell lines were then expressed in the form of $2(-\Delta \Delta \mathrm{Ct})$.

\subsection{Preparation of glioma cell lines lysates}

Cell lines were washed twice with ice-cold phosphate buffered saline (PBS) and scraped into 3-5 volumes of RIPA buffer. After the sonication process for few min, insoluble material was eliminated by centrifuging at $14000 \mathrm{~g}$ for $10 \mathrm{~min}$ at $4^{\circ} \mathrm{C}$. The whole cell lysate was collected and frozen at $-80^{\circ} \mathrm{C}$ before used for protein analysis. The protein concentrations in cell lysates were determined using UV Spectrophotometer.

\subsection{Western immunoblotting analysis}

Cell lysates were subjected to electrophoresis on SDS-polyacrylamide gels and transferred onto nitrocellulose membrane. After blocking this membrane in non-fat dry milk (5\%) in Tris Buffered Saline (TBS) $(10 \mathrm{mM}$ Tris $(\mathrm{pH} 7.5), 150 \mathrm{mM} \mathrm{NaCl})$ for $1 \mathrm{~h}$ at room temperature, membrane was incubated with in primary antibody claudin 3 over night. Blot was again incubated with secondary antibody goat anti-mouse IgG diluted conjugated to alkaline phosphatase (ALP) for one hour at room temperature. Immunoreactivity was done by incubating the blots with BCIP-NBT solution.

\subsection{Statistical analysis}

All continuous variable data derived from sets of gene expression experiments were represented as mean and standard deviation. The relative differences between the gene expression levels were compared with standard two-tailed t-test using InStat Software approach (GraphPad, San Diego, CA). Statistically significant difference between the expression of individual gene expression levels was considered when a $p$ value $<0.05$ was obtained.

\section{FIGURES AND TABLES}

\begin{tabular}{|c|l|c|}
\hline Gene & \multicolumn{1}{|c|}{ Primer sequence } & Product Size \\
\hline Claudin 3-FP & 5'- TCACGTCGCAGAACATCTGG -3' & 236 \\
Claudin 3-RP & 5'-GATGGTGATCTTGGCCTTGG -3' & \\
GAPDH-FP & 5'-TTCGTACCTGGCATTGACTGG-3' & 225 \\
GAPDH-RP & 5'-GAAGGTGAAGGTCGGAGT-3' & \\
\hline
\end{tabular}




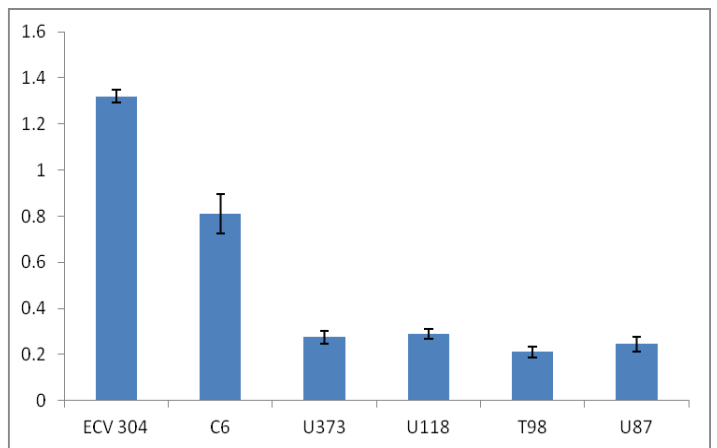

Figure 1: Transcript expression of claudin 3 in glioma cell lines. Claudin 3 expression values were represented in mean and standard deviation for all tumor groups.

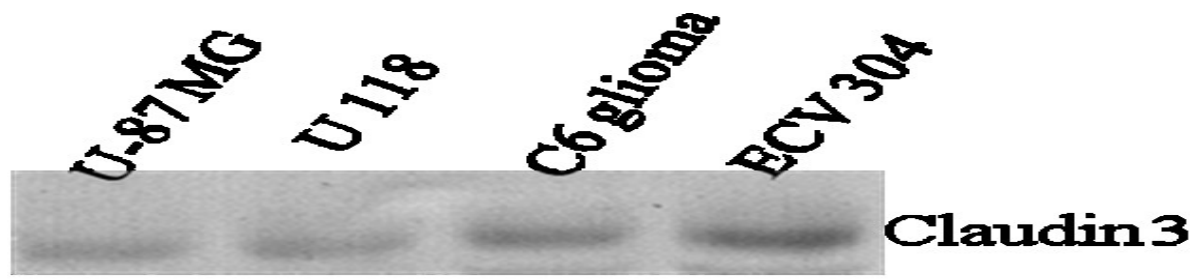

B actin

Fig: 2a) Western blot results of Claudin 3 expression in glioma cell lines.

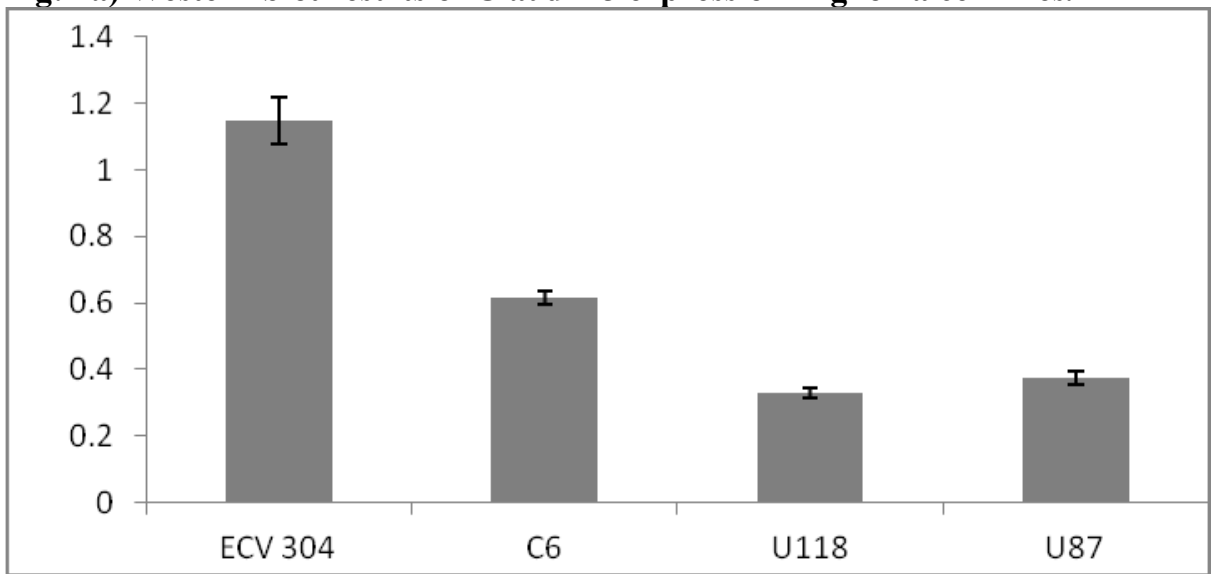

Figure 2b): Protein expression of claudin 3 in glioma cell lines. Claudin 3 expression values were represented in mean and standard deviation for all cell lines.

\section{RESULTS}

In order to examine the claudin 3 expression status in glioma, we have analyzed the gene expression levels of claudin 3 in low grade and high grade glioma cell lines. Figure 1 reveals that, although, a significant down regulation of CDLN3 expression was observed in glioma cell lines of higher grade cell lines i.e. $0.275 \pm$ 0.02 in U373, $0.245 \pm 0.03$ in U87MG, $0.291 \pm 0.02$ in U118, $0.211 \pm 0.02$ in T98 compared to their lower grade counterpart i.e. C6 $(0.81 \pm 0.08)$ and ECV 304 control cell lines $(1.32 \pm 0.02)$.

Western blot analysis results also showed the down regulated expression of claudin 3 protein in high grade cell lines to low grade cell lines. [Fig: 2a\&b]. The results of Real Time PCR and Western blot analysis in glioma cell lines supports the down regulated expression of claudin 3 significantly is associated with high grade glioma progression.

The results of Real Time PCR analysis in low grade and high grade glioma cell lines reveals the down regulated expression of claudin 3 significantly is associated with high grade glioma progression. 


\section{Discussion}

Tight junction proteins are playing important role in barrier and fence functions in epithelial and endothelial cells. Claudin 3 expression was observed in epithelial cells of Liver, pancreas, stomach and intestine [16]. Freeze fracture Studies have shown that claudin 3 of TJs in BBB was associated with either the P- or the E-face of the endothelial cell [17- 19]. Claudin 3 forms TJs associated exclusively with the P-face as is the case for claudin-1, when L-fibroblasts transfected with claudin-3 [20].

In current study we have investigated the expression pattern of tight junction protein claudin 3 in low grade and high grade glioma cell lines. This study was undertaken to understand the biological significance of altered tight junction protein expression in glioma progression. Several studies have reported the CDLN roles in forming TJs [21], conferring ionic selectivity [22] and functioning as a barrier [23], but only few reports have been published on the expression of CDLNs in glioma.

Study by Wolburg et al. shown that maturation of the BBB was not complete at younger age. Younger rats about 3 weeks have developed neutrophil-mediated inflammation in the CNS, whereas older rats have not shown any inflammation at the BBB when these rats were injected LPS [24]. Reports have shown other TJ proteins like claudin-5, occluding expression levels were observed in cerebral vessels of EAE, electron microscopic investigations also supported cerebral vessels were characterized by the presence of inflammatory cells and altered TJ proteins were irregularly formed [24]. Studies have shown detectable proteins levels of claudin-5 and occluding, but claudin 3 expression was absent.

Blood brain barrier leakage and edema formation are important hallmarks of inflammation in the CNS and have been involved in the pathogenesis of EAE [3]. Studies have shown particularly claudin 3 expression was down regulated in glioma as well as in EAE vessels. In our study, Real Time PCR and western blot analysis revealed down regulation of claudin 3 expression in high grade glioma compared to low grade glioma.

\section{Conclusion}

The key finding of the current study is that loss of claudin 3 expression is associated with the progression of malignant gliomas. Our results showed that claudin 3 is down regulated in high grade glioma particularly in glioblastoma multiforme. Results of quantitative Real Time PCR and western blot analysis demonstrated that the expression level of claudin 3 significantly correlates with the World Health Organization histological grades of the glioma. Taken together, these results suggest that claudin 3 is a significant predictor of glioma and could be a potential target for glioma therapy.

\section{Acknowledgements}

We sincerely thank Dr. Ashutosh kumar, University of Hyderabad for guiding Real Time PCR analysis.

\section{REFERENCES}

[1] Bertossi M, Virgintino D, Maiorano E, Occhiogrosso M, Roncali L (1997) Ultrastructural and morphometric investigation of human brain capillaries in normal and peritumoral tissues. Ultrastruct Pathol 21: 41-49

[2] Dinda AK, Sarkar C, Roy S, Kharbanda K, Mathur M, Khosla AK, Banerji AK (1993) A transmission and scanning electron microscopic study of tumoral and peritumoral microblood vessels in human gliomas. J Neurooncol 16: 149-158

[3] Hirano A, Matsui T (1975) Vascular structures in brain tumors. Hum Pathol 6: 611-621

[4] Kniesel U, Wolburg H (2000) Tight junctions of the bloodbrain barrier. Cell Mol Neurobiol 20:57-76

[5] Wolburg H, Lippoldt A (2002) Tight Junctions of the blood brain barrier: development, composition, and regulation. Vasc Pharmacol 38:323-337

[6] DC Davies. Blood-brain barrier breakdown in septic encephalopathy and brain tumours J Anat. 2002 June; $200(6)$ : $639-646$. doi: 10.1046/j.1469-7580.2002.00065.x

[7] Tsukita S, Furuse M, Itoh M (1999) Structural and signalling molecules come together at tight junctions. Curr Opin Cell Biol 11:628-633

[8] Ando-Akatsuka Y, Saitou M, Hirase T, Kishi M, Sakakibara A, Itoh M, Yonemura S, Furuse M, Tsukita S (1996) Interspecies diversity of the occludin sequence: cDNA cloning of human, mouse, dog, and rat-kangaroo homologues. J Cell Biol 133: 43-47

[9] Furuse M, Hirase T, Itoh M, Nagafuchi A, Yonemura S, Tsukita S (1993) Occludin-a novel integral membrane-protein localizing at tight junctions. JCell Biol 123:1777-1788

[10] Furuse M, Fujita K, Hiiragi T, Fujimoto K, Tsukita S (1998) Claudin-1 and -2: novel integral membrane proteins localizing at tight junctions with no sequence similarity to occludin. J CellBiol 141:1539-1550

[11] Morita K, Furuse M, Fujimoto K, Tsukita S (1999) Claudin multigene family encoding four-transmembrane domain protein components of tight junction strands. Proc Natl Acad Sci USA 96:511-516

[12] Furuse, M.; Fujita, K.; Hiiragi, T.; Fujimoto, K.; Tsukita, SH. Claudin-1 and -2: novel integral membrane proteines localizing at tight junctions with no sequence similarity to occludin. $J$ Cell Biol., 1998, 141, 1539-1550.

[13] Morita, K.; Furuse, M.; Fujimoto, K.; Tsukita, S. Claudin multigene family encoding four-transmembrane domain protein components of tight junction strands. Proc Natl Acad Sci USA., 1999, 96(2), 511-516.

[14] Ramirez, SH.; Fan, S.; Dykstra, H.; Rom, S.; Mercer, A.; Reichenbach, NL.; Gofman, L.; Persidsky, Y. Inhibition of Glycogen Synthase Kinase 3b Promotes Tight Junction Stability in Brain Endothelial Cells by Half-Life Extension of Occludin and Claudin-5. PLoS ONE., 2013, 8(2), e55972. [Epub ahead of print].

[15] Furuse M., Sasaki H., Fujimoto K., Tsukita S. A single gene product, claudin-1 or -2 , reconstitutes tight junction strands and recruits occludin in fibroblasts J. Cell Biol. 1431998. 391-401.401b 
[16] Rahner C, Mitic LL, Anderson JM (2001) Heterogeneity in expression and subcellular localization of claudins 2, 3, 4, and 5 in the rat liver, pancreas, and gut. Gastroenterology 120:411-422

[17] Kniesel U, Wolburg H (2000) Tight junctions of the blood brain barrier. Cell Mol Neurobiol 20:57-76

[18] Lippoldt A, Liebner S, Andbjer B, Kalbacher H, Wolburg H, Haller H, Fuxe K (2000) Organization of choroid plexus epithelial and endothelial cell tight junctions and regulation of claudin-1, -2 and -5 expression by protein kinase C. NeuroReport 11:1427-1431

[19] Wolburg H, Lippoldt A (2002) Tight Junctions of the bloodbrain barrier: development, composition, and regulation. VascPharmacol 38:323-337

[20] Furuse M, Sasaki H, Tsukita S (1999) Manner of interaction of heterogeneous claudin species within and between tight junction strands. J Cell Biol 147:891-903

[21] Kniesel, U.; Risau, W.; Wolburg, H. Development of bloodbrain barrier tight junctions in the rat cortex. Dev Brain Res., 1996, 96(1-2), 229-240.

[22] Itoh, M.; Nagafuchi, A.; Yonemura, S.; Kitani-Yasuda, T.; Tsukita, S,; Tsukita, S. The 220 KD protein colocalizing with cadherins in non-epithelial cells is identical to ZO-1, a tight junction associated protein in epithelial cells - cDNA cloning and immunoelectron microscpy. J Cell Biol., 1993, 121(3), 491-502.

[23] Knudsen, KA.; Frankowski, C.; Johnson, KR.; Wheelock MJ. A role for cadherins in cellular signaling and differentiation. $J$ Cell Biochem Suppl., 1998, 30-31, 168-176.

[24] Hartwig Wolburg.; Karen Wolburg-Buchholz.; Jörg Kraus.; Gesa Rascher-Eggstein.; Stefan Liebner.; Stefan Hamm.; Frank Duffner.; Ernst-H. Grote.; Werner Risau.; Britta Engelhardt. Localization of claudin-3 in tight junctions of the blood-brain barrier is selectively lost during experimental autoimmune encephalomyelitis and human glioblastoma multiforme. Acta Neuropathol (2003) 105 : 586-592 DOI 10.1007/s00401-003-0688-Z 